\title{
Persistent Mullerian Duct Syndrome in a Man with Unilateral Cryptorchidism : A Case Report
}

\author{
AZARABADI M*, HEYDARI S, ROUHANI S ,GHANE EZABADI M \\ ${ }^{1}$ Nephrology and Urology Research Center, Baqiyatallah University of Medical Sciences, Tehran, IR Iran \\ ${ }^{*}$ Corresponding author: Mahdi Azarabadi, Nephrology and Urology Research Center, Baqiyatallah University of Medical Sciences, Tehran, \\ Iran. Tel: +98-2181262073, Fax:+98-2181262073, E-mail: Mahdi.azarabadi@gmail.com.
}

\begin{abstract}
introduction: Persistent Mullerian duct syndrome is a rare form of male pseudo-hermaphroditism characterized by the presence of Mullerian duct structures in an otherwise phenotypically, as well asgenotypically, normal man; only a few cases have been reported in the worldwide literature. A greatvariety of organs have been found in indirect inguinal hernial sacs.

Case presentation: We report a case of 28 year old man, father of 2 children with unilateral cryptorchidism on the left side and testis and painful left groin mass. we found uterine tissue extending through the inguinal canal.

Conclusions : PMDS is a rare form of male pseudo-hermaphroditism characterized by the presence of Mullerian duct structures in an otherwise phenotypically, as well as genotypically, normal man. Hernia uteri inguinalis is type I of the male form of PMDS, characterized by one descended testis and the herniation of the ipsilateral corner of the uterus and fallopian tube into the inguinal canal.
\end{abstract}

Keywords: mullerian duct syndrome, cryptorchidism

\section{INTRODUCTION}

Persistent Mullerian duct syndrome (PMDS) was first described by Nilson in 1939.(1) PMID referred to as uterian inguinal hernia, is form of internal male pseudo hermaphroditism.(2) PMDS characterized by the presence of Mullerian duct structures in an otherwise phenotypically, as well as genotypically, normal man.(3) Mullerian derivatives (ie, fallopian tubes, uterus, upper part of the vagina) are present in otherwise normally virilized $X Y$ patients.(4) PMDS patients are both karyotypically and phenotypically male, with normal development of secondary sex characteristics.(5)

\section{CASE REPORT}

A 28 years old man was referred to urology clinic (baghiyatallah hospital) for undesending testis and painful left groin mass. He had a 20 years history of inguinal swelling and absence of left testis. the patient was phenotypically male with normal secondary sexual characters. He was from Zahedan of Iran and had been married for 10 years and having 2 children. Physical examination demonstrated his urethra and penis were fully developed and with a poorly developed left hemiscrotum and no palpable left testis in the scrotum or inguinal canal. The right testis was normally located within the scrotum. There was a non-reducible, tense and tender swelling measuring approximally $8^{*} 6 \mathrm{~cm}$ in the left inguinal region with absent cough impulse. Other examination and routine investigation were normal. CT scan of the abdomen showed a lower abdominal mass at the left side of the urinary bladder.(fig1)Patient was prepared for surgery.

Exploration of the inguinal canal revealed an indirect hernia and an unidentified tubulomascular structure passing from the internal ring could be seen.(fig 2)

Tubular structure resembling uterus .Fallopian tube with an atrophic left testis embedded in the broad ligament and attached to pelvic wall. At this point a decision was made to excise the structure at its point of exit from the inguinal canal

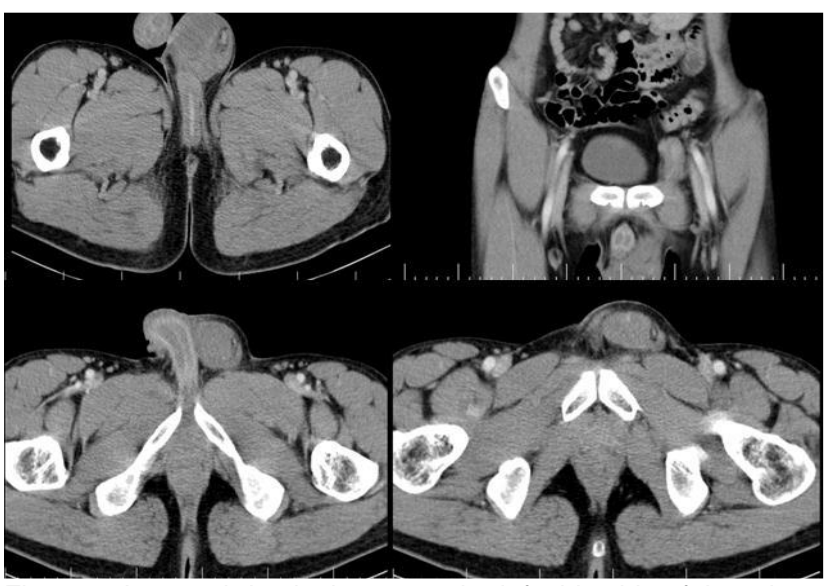

Figure 1: Lower abdominal mass at the left side side of the urinary bladder

No ovarian tissue was seen. Section from left testis showed atrophic seminiferous tubules.no evidence of malignancy was seen. The indirect hernia was repaired using a standard lichtenstien repair with a plug and patch mesh. There were no perioperative complication. On histopathological examination, uterine muscular tissue with its cavity lined by atrophied endometrial tissue was seen .No ovarian tissue was seen. Section from testis showed atrophic seminiferous tubules with hyalinization, sertoli cells.() No evidence of malignancy was seen in tissue sample from testis. Postoperative genotypic analysis were a normal 46XY karyotype. The diagnosis was persistent mullerian duct syndrome (PMDS). 


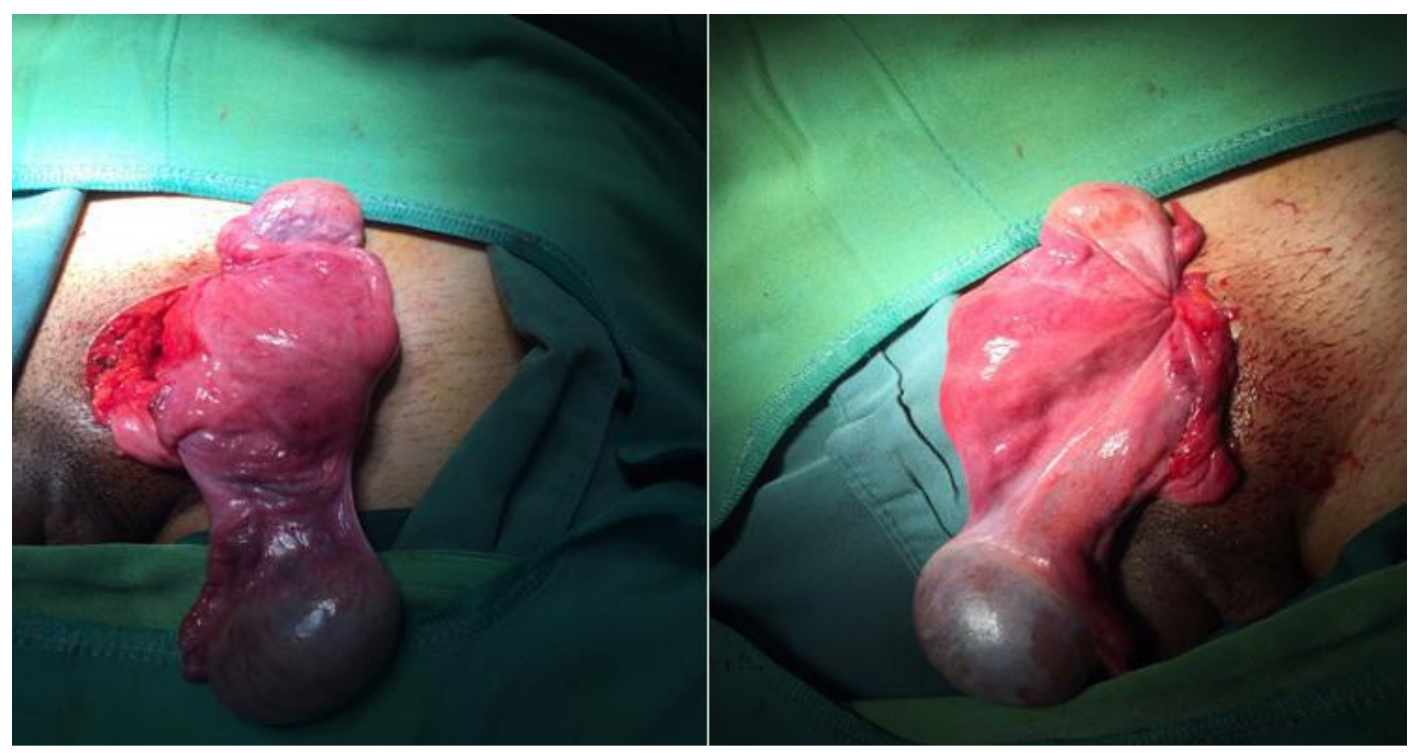

Figure 2 .Intra-operative photograph showing well formed uterus with fallopian tubes and left testes.

\section{DISCUSSION}

Persistent mullerian duct syndrome (PMDS) is a form of internal male pseudohermaphroditism caused by a deficiency of mullerian-inhibiting factor.(6)

Mullerian derivatives (ie, fallopian tubes, uterus, upper part of the vagina) are present in otherwise normally virilized $X Y$ patients. PMDS patients are both karyotypically and phenotypically male, with normal development of secondary sex characteristics. Patients with PMDS usually have normal development of external genitalia and secondary sexual characteristics.(5) The typical patient with PMDS has unilateral or bilateral cryptorchidism and is assigned to the male sex at birth without hesitation, as they have normal male genotypes and phenotypes.(4) Two anatomic variants of PMDS have been described: male and female. The male form is encountered in $80-90 \%$ of cases, characterized by unilateral cryptorchidism with contralateral inguinal hernia, and can be one of the two types: the first type is hernia uteri inguinalis, which is characterized by one descended testis and herniation of the ipsilateral corner of uterus and fallopian tube into the inguinal canal.(7) The second type is crossed testicular ectopia, which is characterized by herniation of both testes and the entire uterus with both fallopian tubes. Clinically, the persistence of a uterus and fallopian tubes leads to either cryptorchidism or inguinal hernia depending on whether or not Mullerian derivatives can be mobilized during testicular descent.(8) If the uterus and fallopian tube are mobile, they may descend into the inguinal canal during testicular descent. How-ever, if the Mullerian structures are relatively immobile testicular descent may be impeded. PMDS is usually coincidently detected during surgical operation, as in our patient's case. However preoperative ultrasonography, computerized tomography and MRI allow possible pre-operative diagnosis.(5)

Management of PMDS is controversial. An element of deciding on intervention is largely based on reducing risk of malignancy, while maintaining maximum reproductive function. In PMDS patients with undescended testes, the rate of testicular cancer is about $12 \%$ which is comparable to the rate seen in unde scended testes not associated with PMDS.(9)

\section{CONCLUSIONS}

PMDS is a rare form of male pseudohermaphroditism characterized by the presence of Mullerian duct structures in an otherwise phenotypically, as well as genotypically, normal man. Since patients are phenotypically male, the diagnosis is usually not suspected until surgery is performed for cryptorchidism or hernia repair. Hernia uteri inguinalis is type I of the male form of PMDS, characterized by one descended testis and the herniation of the ipsilateral corner of the uterus and fallopian tube into the inguinal canal. In order to prevent further complications such as infertility and malignant change, the surgeon should be aware of PMDS while dealing with patients who present with unilateral or bilateral cryptorchidism.

\section{REFERENCES}

1. Nilson O. Hernia uteri inguinalis beim Manne. Acta Chir Scand 1939;83:231.

2. Prakash N, Khurana A, Narula B: Persistent Mullerian duct syndrome.Indian J Pathol Microbiol 2009, 52:546-548

3. Rey R, Picard JY. Embryology and endocrinology of genital development. Baillieres Clin Endocrinol Metab 1998; 12:17-33.

4. Kane GJ. Inguinal hernia containing a paramesonephric (Mullerian) duct in anadult male: a case report. S Afr Med J 1978;53:639.

5. Renu D, Rao BG, Ranganath K, Namitha : Persistent Mullerian duct syndrome. Indian J Radiol Imaging 2010, 20:72-74.

6. Berkmen F. Persistent Müllerian duct syndrome with or without transverse testicular ectopia and testis tumours. $\mathrm{Br} J$ Urol 1997;79:122-6.

7. Gutte AA, Pendharkar PS, Sorte SZ. Transverse testicular ectopia associated with persistent Mullerian duct syndrome - the role of imaging. Br J Radiol 2008;81:e176-8.

8. G kce M, Burgu B, Aydogdu, et al. Transverse testicular ectopia associated with persistent Müllerian duct syndrome: another entity in which magnetic resonance imaging is unreliable. Urology 2010;76:1475-7.

9. Warmann SW, Vogel M, Wehrmann M, et al. Gaint Mullerian duct cyst withmalignant transformation in 15 year old boy. Urology 2006;67:424.

10. Elnemr A, Yonemura $\mathrm{Y}$, Shinbo $\mathrm{M}$, et al. Primary retroperitoneal Mullerian ade-nocarcinoma. Rare Tumors 2010;2(e6):16-9. 